\title{
HOMOTOPY OF PROJECTIONS AND FACTORIZATION IN THE UNITARY GROUP OF CERTAIN $C^{*}$-ALGEBRAS
}

\author{
SHUANG ZHANG
}

\begin{abstract}
.
We prove that every unitary element in the matrix algebra $M_{n}(\mathscr{A})$ and the multiplier algebra $M(\mathscr{A})$ can be factored as a product of a diagonal unitary and some symmetries, where $\mathscr{A}$ is a $C^{*}$-algebra such that $R R(\mathscr{A})=0$ and $\operatorname{tsr}(\mathscr{A})=1$. In addition, we discuss the relations among homotopy of projections, the topological stable rank $\operatorname{tsr}(\mathscr{A})$, and some factorizations of unitary elements in the unitization of $\mathscr{A} \otimes \mathscr{K}$.
\end{abstract}

\section{Introduction.}

Assume that $\mathscr{A}$ is a unital $C^{*}$-algebra, $U(\mathscr{A})$ is the unitary group of $\mathscr{A}$, and $\mathscr{P}(\mathscr{A})$ is the space of all non-trivial projections (i.e., $\neq 0,1$ ) which is equipped with the relative norm topology and called the Grassmann space of $\mathscr{A}$. Despite it is known that $U(\mathscr{A})$ and $\mathscr{P}(\mathscr{A})$ carry a great deal of information about $\mathscr{A}$, their detailed structure, both topological and algebraic, remains mysterious.

The purpose of this article is, first, to consider factorizations of unitaries in a $C^{*}$-algebra $\mathscr{A}$ with $\operatorname{RR}(\mathscr{A})=0$ and $\operatorname{tsr}(\mathscr{A})=1$, in the multiplier algebra $M(\mathscr{A})$ of $\mathscr{A}$ (if $\mathscr{A}$ is non-unital), and in the matrix algebras $M_{n}(\mathscr{A})$ where $n \geqq 1$. It turns out that every unitary element in $M_{n}(\mathscr{A})$ or in $M(\mathscr{A})$ can be factored as a product of two types of simplest unitaries, non-trivial symmetries (self-adjoint unitaries not equal to $+1,-1$ ) and diagonal unitaries. Secondly, we give necessary and sufficient conditions for the surjectivity of the natural map $U_{n}(\mathscr{A}) /$ $U_{n}^{0}(\mathscr{A}) \stackrel{\phi_{n, n+1}}{\longrightarrow} U_{n+1}(\mathscr{A}) / U_{n+1}^{0}(\mathscr{A})$ in terms of homotopy of projections, and consider the same type of factorizations of unitaries in the unitization of $\mathscr{A} \otimes \mathscr{K}$, where $U_{n}(\mathscr{A})$ is the unitary group of $M_{n}(\mathscr{A})$ and $U_{n}^{0}(\mathscr{A})$ is the identity path component of $U_{n}(\mathscr{A})$. All these two goals are reached by considering some intimate relations between the homotopy of projections and factorizations of unitaries.

Supported in part by NSF-DMS9002958 and an NSF Young Investigator grant in the group of Linear Analysis and Probability at the Texas A\&M University.

Received December 3, 1991, in revised form November 2, 1992. 
The factorizations mentioned above provide a better understanding about the unitary groups of $M_{n}(\mathscr{A})$ and the unitization of $\mathscr{A} \otimes \mathscr{K}$ for certain $C^{*}$-algebras. In fact, every symmetry can be written as $2 r-1$ for some projection $r \in \mathscr{P}(\mathscr{A})$ and presumbably well understood, and diagonal unitaries in $M_{n}(\mathscr{A})$ are explicitly created by all unitaries in $U(\mathscr{A})$. However, as one knows, the matrix algebras $M_{n}(\mathscr{A})(n \geqq 1)$ are technically difficult to deal with in general due to the high complexity of the manipulations of matrices with non-commutative entries; in turn it is unknown how $U(\mathscr{A})$ is related to $U_{n}(\mathscr{A})$ if at all.

The text is arranged as follows. In the first section, we prove that two Murray-von Neumann equivalent projections in $\mathscr{A}$ can be conjugated by either one symmetry and a unitary close to the identity in norm or by two symmetries. Based on this result, in the second section we factor each unitary in $U_{n}(\mathscr{A})(n>2)$ as at most $2\left\{\frac{\ln n}{\ln 2}\right\}$ symmetries and a diagonal unitary, where $\{r\}$ is the smallest integer greater or equal to the number $r$. Alternatively, every unitary in $U_{n}(\mathscr{A})$ can be approximated in norm by products of $\left\{\frac{\ln n}{\ln 2}\right\}$ symmetries and a diagonal unitary. Again using the result above, we consider similar factorizations in the unitary group of $M(\mathscr{A})$, for which we need the aids from G. A. Elliott's technique for matroid algebras and our recent result in [59]. It turns out that every unitary in $M(\mathscr{A})$ can be approximated in norm by a product of a symmetry and a diagonal unitary (with respect to a decomposition of the identity); and in case $\mathscr{A}$ is simple, any unitary of $M(\mathscr{A})$ can be factored as a product of $n$ symmetries and a unitary of the form $\left(1-p_{n}\right) \oplus\left(p_{n} u_{0} p_{n}\right)$ for some projection $p_{n} \in M(\mathscr{A})$ such that $2^{n}\left[p_{n}\right]=[1]$ for any $n \geqq 2$.

In the last section, assuming that $\mathscr{A}$ is an arbitrary unital $C^{*}$-algebra, we discuss the relation between the homotopy of projections and factoring unitaries in $U_{n}(\mathscr{A})$ as a product of symmetries and a diagonal unitary. In particular, the natural map

$$
U_{n}(\mathscr{A}) / U_{n}^{0}(\mathscr{A}) \stackrel{\phi_{n, \infty}}{\longrightarrow} K_{1}(\mathscr{A})
$$

is surjective if for each $m>n$ the path component of the space of projections in $M_{m}(\mathscr{A})$ containing the projection $1 \otimes e_{11}$ is connected, where $1 \otimes e_{11}$ is the $m \times m$ matrix whose only nonzero entry 1 is at the (1,1)-place. In case the topological stable rank $n:=\operatorname{tsr}(\mathscr{A})$ of $\mathscr{A}$ is finite, every unitary in the unitization of $\mathscr{A} \otimes \mathscr{K}$, whose unitary group is denoted by $U_{\infty}(\mathscr{A})$, can be approximated in norm by some products of a number of symmetries and a unitary of $U_{n}(\mathscr{A})$ (embedded into $U_{\infty}(\mathscr{A})$ ), where $\mathscr{K}$ denotes the algebra of all compact operators 
on a separable Hilbert space; consequently, $\mathscr{A} \otimes \mathscr{K}$ is generated by projections and unitaries in $U_{m}(\mathscr{A})$ for some $m>n$.

ACKNOWLEDGMENT. The author wishes to thank the referee for his/her extremely helpful suggestions and detailed criticisms for improving the manuscript.

\section{Factorizations in $U(\mathscr{A})$.}

\subsection{Matrix decomposition of a $C^{*}$-algebra.}

Let $\left\{p_{i}\right\}$ be a set of mutually orthogonal nonzero projections of $\mathscr{A}$ such that

$$
\sum_{i} \oplus p_{i}=1
$$

here we assume that such projections exist in $\mathscr{A}$. The above sum is called a decomposition of the identity with respect to $\left\{p_{i}\right\}$. Suppose further that the above decomposition contains a finite number of terms, if $\mathscr{A}$ is unital, and it contains infinitely many terms converging to the identity of $M(\mathscr{A})$ in the strict topology, if $\mathscr{A}$ is non-unital. We will often use the following ${ }^{*}$-isomorphism between a $C^{*}$-algebra $\mathscr{A}($ or $M(\mathscr{A})$ ) and a matrix decomposition of $\mathscr{A}($ or $M(\mathscr{A})$, respectively) with respect to the decomposition $\sum_{i} p_{i}=1$ :

$$
x \mapsto\left(\begin{array}{cccc}
p_{1} x p_{1} & p_{1} x p_{2} & p_{1} x p_{3} & \ldots \\
p_{2} x p_{1} & p_{2} x p_{2} & p_{2} x p_{3} & \ldots \\
p_{3} x p_{1} & p_{3} x p_{2} & p_{3} x p_{3} & \ldots \\
\vdots & \vdots & \vdots & \ddots
\end{array}\right) .
$$

Under this *-isomorphism, one can easily check that the multiplication, the addition, the scalar multiplication and the involution follow the standard rules of the matrix manipulations. The above matrix is said to be the matrix form of $x$ with respect to the decomposition $\sum_{i} p_{i}=1$.

\subsection{Notations.}

Let $\mathscr{A}$ be a unital $C^{*}$-algebra and $U(\mathscr{A})$ be equipped with the relative norm topology. Two unitaries in $U(\mathscr{A})$ are said to be homotopic if they are in the same path component of $U(\mathscr{A})$. Slightly abusing a standard notation, we use $\tilde{\mathscr{A}}$ to denote the unitization of $\mathscr{A}$ in case $\mathscr{A}$ is non-unital and to denote $\mathscr{A}$ itself in case $\mathscr{A}$ is unital.

As usual, the notation ' $p \sim q$ ' denotes the Murray-von Neumann equivalence in $\mathscr{P}(\mathscr{A})$, and $[p]$ denotes the equivalence class containing $p$. Under the natural ordering induced by the equivalence, $[p] \leqq[q]$ iff $p$ is equivalent to a subprojection of $q$.

Two projections $p, q \in \mathscr{P}(\mathscr{A})$ are said to be unitarily equivalent, if there exists 
a unitary $u$ of $\tilde{\mathscr{A}}$ such that $u p u^{*}=q$; and $p$ and $q$ are said to be homotopic if $p$ and $q$ are in the same path component of $\mathscr{P}(\mathscr{A})$. In case $\operatorname{RR}(\mathscr{A})=0$ and $\operatorname{tsr}(\mathscr{A})=1$, two projections $p, q \in \mathscr{P}(\mathscr{A})$ are equivalent if and only if $p$ and $q$ are unitarily equivalent, again if and only if $p$ and $q$ are homotopic [48, 3.4]). The same conclusions hold also for purely infinite simple $C^{*}$-algebras ([21] and [51]).

A $C^{*}$-algebra is said to have the cancellation property, if $p_{1} \sim p_{2}$.whenever four projections of $\mathscr{A}$ satisfy $r_{1} \oplus p_{1} \sim r_{2} \oplus p_{2}$ and $r_{1} \sim r_{2}$. Under the condition $\operatorname{RR}(\mathscr{A})=0, \mathscr{A}$ has cancellation if and only if $\operatorname{tsr}(\mathscr{A})=1$ ([3]). Specific examples of such $C^{*}$-algebras include the following $C^{*}$-algebras and the tensor products with the algebra $\mathscr{K}$ : all AF algebras [20], all Bunce-Deddens algebras ([7], [6]), all finite factors (von Neumannalgebras) [13], many inductive limit algebras ([4], [23], [5], among others), and certain irrational rotation algebras ([41], [14]).

1.3. Lemma. Assume that $\operatorname{RR}(\mathscr{A})=0$ and $\operatorname{tsr}(\mathscr{A})=1$. Then the following three statements are equivalent:

(i) Two projections $p, q \in \mathscr{P}(\mathscr{A})$ are equivalent.

(ii) For each positive number $\varepsilon$ there exists a symmetry s and a unitary $u_{\varepsilon}$ in $\tilde{\mathscr{A}}$ such that $\left\|u_{\varepsilon}-1\right\|<\varepsilon$ and $s u_{\varepsilon} p u_{\varepsilon}^{*} s=q$.

(iii) There are two symmetries $s$ and $s^{\prime}$ in $\tilde{\mathscr{A}}$ such that $s s^{\prime} p s^{\prime} s=q$.

Proof. It is trivial that (iii) implies (1). In order to prove that (i) implies (ii) and (ii) implies (iii), we need to use the construction in $[48,2.1]$ which is based on the idea in [11] with further analyses. First, write $p$ as a $2 \times 2$ matrix form with respect to the decomposition $q \oplus(1-q)=1$, that is,

$$
p=\left(\begin{array}{cc}
q p q & q p(1-q) \\
(1-q) p q & (1-q) p(1-q)
\end{array}\right)
$$

For each positive number $\delta<1-\frac{1}{\sqrt{2}}$ there exists $[48,2.1]$ a projection $p_{0}$ with a matrix form

$$
p_{0}=\left(\begin{array}{ll}
a^{\prime} & b \\
b^{*} & c^{\prime}
\end{array}\right)
$$

such that

$$
\left\|p-p_{0}\right\|<\delta
$$

where $a^{\prime}$ and $c^{\prime}$ are positive elements of $\mathscr{A}$ with finite spectra in $[0,1]$. If $\delta$ is small enough, it is well known that there exists a unitary $u_{\varepsilon} \in U(\mathscr{A})$ such that

$$
\left\|u_{\varepsilon}-1\right\|<\varepsilon \text { and } u_{\varepsilon} p u_{\varepsilon}^{*}=p_{0} .
$$

Set $f_{0}=\chi_{\{1\}}\left(a^{\prime}\right)$ and $e_{0}=\chi_{\{1\}}\left(c^{\prime}\right)$ (it is possible that $f_{0}=0$ or $e_{0}=0$, or both are 
zero). Of course $f_{0}$ is a projection in $q \mathscr{A} q$ and $e_{0}$ is a projection in $(1-q) \mathscr{A}(1-q)$. With respect to the decomposition $f_{0} \oplus\left(q-f_{0}\right)=q$ one can write

$$
a^{\prime}=\left(\begin{array}{cc}
f_{0} & 0 \\
0 & a
\end{array}\right)
$$

where $\sigma(a) \backslash\{0\}:=\left\{t_{i}\right\}_{i=1}^{m} \subset(0,1)$. Similarly, with respect to $\left(1-q-e_{0}\right) \oplus e_{0}=$ $1-q$ one can write

$$
c^{\prime}=\left(\begin{array}{cc}
c & 0 \\
0 & e_{0}
\end{array}\right)
$$

Then $\sigma(c) \backslash\{0\}=\left\{1-t_{i}\right\}_{i=1}^{m} \subset(0,1)$, since

$$
\left(\begin{array}{cc}
a & b \\
b^{*} & c
\end{array}\right)
$$

is a subprojection of $p_{0}$, where one notices that $f_{0} b=0$ and $b e_{0}=0$ due to the equalities $a-a^{2}=b b^{*}$ and $c-c^{2}=b^{*} b$. Thus, with respect to the decomposition

$$
f_{0} \oplus\left(q-f_{0}\right) \oplus\left(1-q-e_{0}\right) \oplus e_{0}=1
$$

one can write $p_{0}$ as the following $4 \times 4$ matrix form:

$$
p_{0}=\left(\begin{array}{cccc}
f_{0} & 0 & 0 & 0 \\
0 & a & b & 0 \\
0 & b^{*} & c & 0 \\
0 & 0 & 0 & e_{0}
\end{array}\right) ;
$$

where $a=\left(q-f_{0}\right) p_{0}\left(q-f_{0}\right), b=\left(q-f_{0}\right) p_{0}\left(1-q-e_{0}\right)$, and $c=\left(1-q-e_{0}\right)$ $p_{0}\left(1-q-e_{0}\right)$. By the construction in [48, 2.1], if one writes

$$
c=\sum_{i=1}^{m}\left(1-t_{i}\right) r_{i}
$$

for some mutually orthogonal projections $\left\{r_{i}\right\}_{i=1}^{m} \subset\left(1-q-e_{0}\right) \mathscr{A}\left(1-q-e_{0}\right)$, then

$$
a=v\left(\sum_{i=1}^{m} r_{i}-c\right) v^{*}=\sum_{i=1}^{m} t_{i} v r_{i} v^{*}
$$

for some partial isometry $v \in \mathscr{A}$ such that $v^{*} v=\sum_{i=1}^{m} r_{i}:=e$. Furthermore, the relation among $b, c$ and $v$ is given by

$$
b=v(e-c)^{\frac{1}{2}} . .
$$

Set $v v^{*}=f$. Clearly, ef $=0, f$ is the range projection of $a$, and $e$ is the range 
projection of $c$. Obviously, $f_{0} f=0$ and $e_{0} e=0$. It is due to the cancellation property that $q-f_{0}-f \neq 0$ as long as $e_{0} \neq 0$.

Since $\sigma(a) \cup \sigma(c) \subset[0,1)$, it is obvious that

$$
\begin{aligned}
& \|a\|<1, \quad\|f-a\|<1, \\
& \|c\|<1, \quad\|e-c\|<1 .
\end{aligned}
$$

It follows from the well known fact (we cannot locate a reference)

$$
\left\|q_{1}-q_{2}\right\|=\max \left\{\left\|q_{1}\left(1-q_{2}\right)\right\|,\left\|q_{2}\left(1-q_{1}\right)\right\|\right\}
$$

that

$$
\left\|\left(\begin{array}{cc}
a & b \\
b^{*} & c
\end{array}\right)-f\right\|<1, \quad\left\|\left(\begin{array}{cc}
a & b \\
b^{*} & c
\end{array}\right)-e\right\|<1 .
$$

Set

$$
s_{0}=\left\{e \oplus f-\left(\left(\begin{array}{cc}
a & b \\
b^{*} & c
\end{array}\right)-f\right)^{2}\right\}^{-\frac{1}{2}}\left\{\left(\begin{array}{cc}
a & b \\
b^{*} & c
\end{array}\right)+f-(e \oplus f)\right\} .
$$

Then $s_{0}$ is a symmetry of $(e \oplus f) \mathscr{A}(e \oplus f)$ such that

$$
s_{0} f s_{0}=\left(\begin{array}{cc}
a & b \\
b^{*} & c
\end{array}\right)
$$

One can also similarly define a symmetry conjugating $\left(\begin{array}{cc}a & b \\ b^{*} & c\end{array}\right)$ and $e$, but we do not need it here.

Let us show now that (i) implies (ii). If $q \sim p$, it follows from the cancellation property that $q-f_{0}-f \sim e_{0}$, for $p \sim p_{0}$. Let $v_{0}$ be a partial isometry in $\mathscr{A}$ such that $v_{0} v_{0}^{*}=e_{0}$ and $v_{0}^{*} v_{0}=q-f_{0}-f$. Since $e_{0}\left(q-f_{0}-f\right)=0$, then $s_{1}:=v_{0}+$ $v_{0}^{*}$ is a symmetry in the corner

$$
\left(e_{0} \oplus\left(q-f_{0}-f\right)\right) \mathscr{A}\left(e_{0} \oplus\left(q-f_{0}-f\right)\right)
$$

such that $s_{1}\left(q-f_{0}-f\right) s_{1}=e_{0}$. Set

$$
s:=s_{0} \oplus s_{1} \oplus f_{0} \oplus\left(1-q-e-e_{0}\right) .
$$

Then $s$ is a symmetry of $\tilde{\mathscr{A}}$ such that $s p_{0} s=q$. Therefore, $s u_{\varepsilon} p u_{\varepsilon}^{*} s=q$, and hence (ii) holds.

For (ii) implying (iii), we need to find a symmetry $s^{\prime} \in \tilde{\mathscr{A}}$ such that $s^{\prime} p s^{\prime}=u_{\varepsilon} p u_{\varepsilon}^{*}$. The existence of $s^{\prime}$ follows immediately from the estimate $\left\|p-u_{\varepsilon} p u_{\varepsilon}^{*}\right\| \leqq$ $2\left\|u_{\varepsilon}-1\right\|<1$ (if $\varepsilon<\frac{1}{2}$ ) and a well known fact that two close projections are equivalent by a symmetry (for example, see [61, Lemma 2]).

We now have the following factorization result in $U(\tilde{\mathscr{A}})$. 
1.4. THEOREM. Suppose that $\operatorname{RR}(\mathscr{A})=0, \operatorname{tsr}(\mathscr{A})=1, p$ is any nonzero projection of $\mathscr{A}, u$ is an arbitrary unitary in $\tilde{A}$, and $\varepsilon$ is any positive number.

(i) There exist two symmetries $s, s^{\prime}$ and a unitary $u_{\varepsilon}$ in $\tilde{\mathscr{A}}$ such that

$$
\left\|u_{\varepsilon}-1\right\|<\varepsilon \text { and } u=u_{\varepsilon} s\left(\begin{array}{cc}
u_{1} & 0 \\
0 & u_{2}
\end{array}\right)=s s^{\prime}\left(\begin{array}{cc}
u_{1}^{\prime} & 0 \\
0 & u_{2}^{\prime}
\end{array}\right)
$$

where $u_{1}$ and $u_{1}^{\prime}$ are unitaries of $p \mathscr{A} p, u_{2}$ and $u_{2}^{\prime}$ are unitaries of $(1-p) \tilde{\mathscr{A}}(1-p)$.

(ii) If $q \in \mathscr{P}(\mathscr{A})$ with $p \sim q \leqq 1-p$, then there exist two symmetries $s$, $s^{\prime}$ and a unitary $u_{\varepsilon}$ of $\tilde{\mathscr{A}}$ such that $\left\|u_{\varepsilon}-1\right\|<\varepsilon$ and

$$
\begin{aligned}
u & =u_{\varepsilon} s\left(\begin{array}{ll}
0 & a \\
b & c
\end{array}\right)=u_{\varepsilon} s\left(\begin{array}{cc}
0 & b^{*} \\
b & c c^{*}
\end{array}\right)\left(\begin{array}{cc}
p & 0 \\
0 & b a+c
\end{array}\right) \\
& =s s^{\prime}\left(\begin{array}{ll}
0 & a^{\prime} \\
b^{\prime} & c^{\prime}
\end{array}\right)=s s^{\prime}\left(\begin{array}{cc}
0 & b^{\prime *} \\
b^{\prime} & c^{\prime} c^{*}
\end{array}\right)\left(\begin{array}{cc}
p & 0 \\
0 & b^{\prime} a^{\prime}+c^{\prime}
\end{array}\right),
\end{aligned}
$$

where the matrices above are unitaries of $\tilde{\mathscr{A}}$ (with respect to the decomposition $p \oplus(1-p)=1)$, and

$$
\left(\begin{array}{cc}
0 & b^{*} \\
b & c c^{*}
\end{array}\right) \text { and }\left(\begin{array}{cc}
0 & b^{*} \\
b^{\prime} & c^{\prime} c^{*}
\end{array}\right)
$$

are symmetries of $\tilde{\mathscr{A}}$.

ProOF. (i) Since $u p u^{*}$ is unitarily equivalent to $p$, by Lemma (1.3) there exist two symmetries $s, s^{\prime}$ and a unitary $u_{\varepsilon}$ close to the identity (in norm) within $\varepsilon$ such that

$$
s u_{\varepsilon}^{*} u p=p s u_{\varepsilon}^{*} u \text { and } \quad s^{\prime} s u p=p s^{\prime} s u
$$

It follows that

$$
u=u_{\varepsilon} s\left(\begin{array}{cc}
u_{1} & 0 \\
0 & u_{2}
\end{array}\right)=s s^{\prime}\left(\begin{array}{cc}
u_{1}^{\prime} & 0 \\
0 & u_{2}^{\prime}
\end{array}\right)
$$

(ii) If $p \sim q \leqq 1-p$, then $u p u^{*} \sim q$. It follows from Lemma (1.3) again that

$$
s u_{\varepsilon}^{*} u p u^{*} u_{\varepsilon} s=s^{\prime} s u p u^{*} s s^{\prime}=q,
$$

where $s$ and $s^{\prime}$ are symmetries and $u_{\varepsilon}$ is a unitary close to 1 in norm within $\varepsilon$. It is then easily checked by calculation (see $[55,2,1]$, if not clear) that

$$
s u_{\varepsilon}^{*} u=\left(\begin{array}{ll}
0 & a \\
b & b
\end{array}\right) \text { and } s^{\prime} s u=\left(\begin{array}{cc}
0 & a^{\prime} \\
b^{\prime} & c^{\prime}
\end{array}\right)
$$

while by matrix multiplication $([55,2.1])$ one sees 


$$
\begin{aligned}
& \left(\begin{array}{ll}
0 & a \\
b & c
\end{array}\right)=\left(\begin{array}{cc}
0 & b^{*} \\
b & c c^{*}
\end{array}\right)\left(\begin{array}{cc}
p & 0 \\
0 & b a+c
\end{array}\right) \text { and } \\
& \left(\begin{array}{cc}
0 & a^{\prime} \\
b^{\prime} & c^{\prime}
\end{array}\right)=\left(\begin{array}{cc}
0 & b^{*} \\
b^{\prime} & c^{\prime} c^{*}
\end{array}\right)\left(\begin{array}{cc}
p & 0 \\
0 & b^{\prime} a^{\prime}+c^{\prime}
\end{array}\right) .
\end{aligned}
$$

Thus, we have completed the proof.

\subsection{REMARKS.}

(i) Some conclusions similar to Theorem 1.4 also hold in case $\mathscr{A}$ is a purely infinite, simple $C^{*}$-algebra; namely, there exist two symmetries $s, s^{\prime}$ of $\tilde{\mathscr{A}}$ such that

$$
u=s^{\prime} s\left(\begin{array}{cc}
u_{1} & 0 \\
0 & u_{2}
\end{array}\right)
$$

where $u_{1}$ is a unitary of $(1-p) \tilde{A}(1-p)$, and $u_{2}$ is a unitary of $p \mathscr{A} p$; alternatively, there exist symmetries $s_{1}$ and $s_{2}$ of $\tilde{\mathscr{A}}$ such that

$$
u=s_{2} s_{1}\left(\begin{array}{ll}
0 & a \\
b & c
\end{array}\right)=s_{2} s_{1}\left(\begin{array}{cc}
0 & b^{*} \\
b & c c^{*}
\end{array}\right)\left(\begin{array}{cc}
1-p & 0 \\
0 & b a+c
\end{array}\right)
$$

the above matrix decompositions are with respect to $p \oplus(1-p)=1$. Since purely infinite simple $C^{*}$-algebras are not the target of this article, we leave a proof for the above claim elsewhere $([61,1.5])$.

(ii) We can write the unitary $u$ in Theorem 1.4 as a product in the following form

$$
u=\left(u_{\varepsilon} s u_{\varepsilon}^{*}\right) u_{\varepsilon}\left(\begin{array}{cc}
u_{1} & 0 \\
0 & u_{2}
\end{array}\right), \quad u=\left(u_{\varepsilon} s u_{\varepsilon}^{*}\right) u_{\varepsilon}\left(\begin{array}{ll}
0 & a \\
b & c
\end{array}\right)
$$

where $u_{\varepsilon} s u_{\varepsilon}^{*}$ is also a symmetry. Hence, the order of $s$ and $u_{\varepsilon}$ in the above factorizations is up to one's choice. This observation will be freely used later without further comments.

\section{Factorization of unitaries in $M_{n}(\mathscr{A})$.}

Applying the factorizations theorem (1.4) in the last section, we will factor unitaries in $M_{n}(\tilde{\mathscr{A}})$ as a product of some symmetries and a diagonal unitary in this section. Using the elementary fact that each symmetry can be written as $2 r-1$ for some projections, one sees that the construction of $U_{n}(\mathscr{A})$ is completely determined by the Grassmann space of $M_{n}(\mathscr{A})$ and unitary of $\mathscr{A}$ itself.

\subsection{Further Notations.}

From now on, the identity of $M_{n}(\tilde{A})$ is denoted by $I_{n}$ which is often written as

$$
\sum_{i=1}^{n} 1 \otimes e_{i i}=I_{n}
$$


where $\left\{1 \otimes e_{i j}\right)$, denotes the set of matrices whose only nonzero entry 1 is at the $(i, j)$-place.

2.2. TheOREM. Suppose that $\mathscr{A}$ is a $C^{*}$-algebra such that $\mathrm{RR}(\mathscr{A})=0$ and $\operatorname{tsr}(\mathscr{A})=1$. If $p=p_{1} \oplus p_{2} \oplus \ldots \oplus p_{n}$ for some projections $\left\{p_{i}\right\} \subset \mathscr{P}(\mathscr{A})$ such that $\left[p_{1}\right] \leqq\left[p_{2}\right] \leqq \ldots \leqq\left[p_{n}\right]$ (i.e., $p_{i} \sim q_{i} \leqq p_{i+1}$ for $\left.1 \leqq i \leqq n-1\right)$ and $u$ is a unitary of the corner $p \mathscr{A} p$, then there exist some symmetries $\left\{s_{i}\right\}_{i=1}^{m},\left\{s_{j}^{\prime}\right\}_{j=1}^{2 m},\left\{x_{i}\right\}_{i=1}^{2 m}$ and $\left\{y_{j}\right\}_{j=1}^{3 m}$ of $p \mathscr{A} p$ such that $u$ can be approximated in norm by unitaries of $p \mathscr{A} p$ with both the following matrix forms

$$
s_{1} s_{2} \ldots s_{m}\left(\begin{array}{cccc}
u_{1} & 0 & \ldots & 0 \\
0 & u_{2} & \ddots & \vdots \\
\vdots & \ddots & \ddots & 0 \\
0 & \ldots & 0 & u_{n}
\end{array}\right), s_{1}^{\prime} s_{2}^{\prime} \ldots s_{2 m}^{\prime}\left(\begin{array}{cccc}
p_{1} & 0 & \ldots & 0 \\
0 & \ddots & \ddots & \vdots \\
\vdots & \ddots & p_{n-1} & 0 \\
0 & \ldots & 0 & v
\end{array}\right)
$$

and $u$ can be exactly factored as the following products

$$
u=x_{1} x_{2} \ldots x_{2 m}\left(\begin{array}{cccc}
u_{1}^{\prime} & 0 & \ldots & 0 \\
0 & u_{2}^{\prime} & \ddots & \vdots \\
\vdots & \ddots & \ddots & 0 \\
0 & \ldots & 0 & u_{n}^{\prime}
\end{array}\right)=y_{1} y_{2} \ldots y_{3 m}\left(\begin{array}{cccc}
p_{1} & 0 & \ldots & 0 \\
0 & \ddots & \ddots & \vdots \\
\vdots & \ddots & p_{n-1} & 0 \\
0 & \ldots & 0 & v^{\prime}
\end{array}\right) \text {, }
$$

where $u_{i}$ and $u_{i}^{\prime}$ are some unitaries of the corner $p_{i} \mathscr{A} p_{i}(1 \leqq i \leqq n)$, $v$ and $v^{\prime}$ are some unitaries of $p_{n} \mathscr{A} p_{n}$, and $m$ is the smallest integer such that $2^{m} \geqq n$.

As a consequence, if $\mathscr{A}$ is replaced by $M_{n}(\tilde{\mathscr{A}})$, p by $I_{n}$, and $p_{i}$ by $1 \otimes e_{i i}$, then every unitary u of $M_{n}(\tilde{\mathscr{A}})$ can be approximated by products of some ( $m$ or $2 m$ ) symmetries and a diagonal unitary (in either of the above two forms) and can be exactly factored as products of some $(2 \mathrm{~m}$ or $3 \mathrm{~m}$ ) symmetries and a diagonal unitary (in either of the above two forms).

Proof. The proof is given by repeatedly using the factorizations in Theorem 1.4 .

First, for any $\varepsilon>0$ we carry out the following two factorizations

$$
u=s_{1} s_{2} \ldots s_{m} u_{\varepsilon}\left(\begin{array}{cccc}
u_{1} & 0 & \ldots & 0 \\
0 & u_{2} & \ddots & \vdots \\
\vdots & \ddots & \ddots & 0 \\
0 & \ldots & 0 & u_{n}
\end{array}\right)=x_{1} x_{2} \ldots x_{2 m}\left(\begin{array}{cccc}
u_{1}^{\prime} & 0 & \ldots & 0 \\
0 & u_{2}^{\prime} & \ddots & \vdots \\
\vdots & \ddots & \ddots & 0 \\
0 & \ldots & 0 & u_{n}^{\prime}
\end{array}\right) \text {, }
$$

where $\left\|u_{\varepsilon}-p\right\|<\varepsilon$.

Case 1. Assume that $n=2^{m}$ for some integer $m$.

This is an elementary induction argument on $m$. Let $q=p_{1} \oplus p_{2} \oplus \ldots \oplus p_{2^{m-1}}$. Then $p-q=p_{2^{m-1}+1} \oplus \ldots \oplus p_{n}$. Since $u q u^{*}$ and $q$ are unitarily equivalent, by 
Theorem 1.4. (i) there exists some symmetries $s_{1}\left(=x_{1}\right), s_{2}$, and a unitary $v_{1}$ in $p \mathscr{A} p$ such that $\left\|v_{1}-p\right\|<\varepsilon$, and

$$
s_{1} v_{1} u=\left(\begin{array}{cc}
u_{1} & 0 \\
0 & w_{1}
\end{array}\right) \text { and } s_{2} s_{1} u=\left(\begin{array}{cc}
u_{1}^{\prime} & 0 \\
0 & w_{1}^{\prime}
\end{array}\right)
$$

where $u_{1}, u_{1}^{\prime}$ are unitaries of $q \mathscr{A} q$ and $w_{1}, w_{1}^{\prime}$ are unitaries of $(p-q) \mathscr{A}(p-q)$. Now the induction applies to the corners of both unitaries

$$
\left(\begin{array}{cc}
u_{1} & 0 \\
0 & w_{1}
\end{array}\right) \text { and }\left(\begin{array}{cc}
u_{1}^{\prime} & 0 \\
0 & w_{1}^{\prime}
\end{array}\right) \text {. }
$$

There are some symmetries $s_{2}^{\prime}, s_{3}^{\prime}, \ldots, s_{m}^{\prime}, x_{3}^{\prime}, x_{4}^{\prime}, \ldots, x_{2 m}^{\prime}$ and a unitary $v_{2}^{\prime}$ of $q \mathscr{A} q$ close to $q$ in norm such that

$$
\begin{gathered}
u_{1}=s_{2}^{\prime} s_{3}^{\prime} \ldots s_{m}^{\prime} v_{2}^{\prime}\left(\begin{array}{cccc}
u_{1} & 0 & \ldots & 0 \\
0 & u_{2} & \ddots & \vdots \\
\vdots & \ddots & \ddots & 0 \\
0 & 0 & 0 & u_{2^{m-1}}
\end{array}\right) \text { and } \\
u_{1}^{\prime}=x_{3}^{\prime} x_{4}^{\prime} \ldots x_{2 m}^{\prime}\left(\begin{array}{cccc}
u_{1}^{\prime} & 0 & \ldots & 0 \\
0 & u_{2}^{\prime} & \ddots & \vdots \\
\vdots & \ddots & \ddots & 0 \\
0 & 0 & 0 & u_{2^{m-1}}^{\prime}
\end{array}\right)
\end{gathered}
$$

and there are some symmetries $s_{2}^{\prime \prime}, s_{3}^{\prime \prime}, \ldots, s_{m}^{\prime \prime}, x_{3}^{\prime \prime}, x_{4}^{\prime \prime}, \ldots, x_{2 m}^{\prime \prime}$ and a unitary $v_{2}^{\prime}$ of $(p-q) \mathscr{A}(p-q)$ close to $p-q$ in norm such that

$$
\begin{gathered}
w_{1}=s_{2}^{\prime \prime} s_{3}^{\prime \prime} \ldots s_{m}^{\prime \prime} v_{2}^{\prime}\left(\begin{array}{cccc}
u_{2^{m-1}+1} & 0 & \ldots & 0 \\
0 & u_{2^{m-1}+2} & \ddots & \vdots \\
\vdots & \ddots & \ddots & 0 \\
0 & \ldots & 0 & u_{2^{m}}
\end{array}\right) \text { and } \\
w_{1}^{\prime}=x_{1} x_{2} \ldots x_{2 m}\left(\begin{array}{cccc}
u_{2 m-1+1}^{\prime} & 0 & \ldots & 0 \\
0 & u_{2^{m-1}+2}^{\prime} & \ddots & \vdots \\
\vdots & \ddots & \ddots & 0 \\
0 & \ldots & 0 & u_{2^{m}}^{\prime}
\end{array}\right) \text {. }
\end{gathered}
$$

For each $i \geqq 2$ set

$$
s_{i}=\left(\begin{array}{cc}
s_{i}^{\prime} & 0 \\
0 & s_{i}^{\prime \prime}
\end{array}\right), \quad x_{i}=\left(\begin{array}{cc}
x_{i}^{\prime} & 0 \\
0 & x_{i}^{\prime \prime}
\end{array}\right), \quad \text { and } \quad v_{2}=\left(\begin{array}{cc}
v_{2}^{\prime} & 0 \\
0 & v_{2}^{\prime \prime}
\end{array}\right)
$$

Thus, for the case $n=2^{m}$ the two factorizations follow clearly, where one needs to notice the observation in (ii) of (1.5). 
Case 2. Assume that $n<2^{m}$, where $m$ is the least integer satisfying this inequality.

Applying the same arguments as in the case 1 to the projection $q=\oplus_{i=1}^{2^{m-1}} p_{i}$ and any unitary $u$ of $p \mathscr{A} p$, we find two symmetries $s_{0}, s_{0}^{\prime}$ and a unitary $v_{0}$ in $p \mathscr{A} p$ such that

$$
u=s_{0} v_{0}\left(\begin{array}{cc}
w_{1} & 0 \\
0 & w_{2}
\end{array}\right)=s_{0} s_{0}^{\prime}\left(\begin{array}{cc}
w_{1}^{\prime} & 0 \\
0 & w_{2}^{\prime}
\end{array}\right)
$$

where the matrix forms are with respect to the decomposition

$$
\left(\oplus_{i=1}^{2^{m-1}} p_{i}\right) \oplus\left(\oplus_{i=2^{m-1}+1}^{n} p_{i}\right)=p .
$$

Notice that $1<n-2^{m-1}<2^{m-1}$. The two factorizations of $u$ clearly follow from the same arguments as for the case 1 applied to the corners corners $w_{1}, w_{2}$, $w_{1}^{\prime}$, and $w_{2}^{\prime}$.

Secondly, we carry out the factorization

$u=s_{1}^{\prime} s_{2}^{\prime} \ldots s_{2 m}^{\prime} u_{\varepsilon}^{\prime}\left(\begin{array}{cccc}p_{1} & 0 & \ldots & 0 \\ 0 & \ddots & \ddots & \vdots \\ \vdots & \ddots & p_{n-1} & 0 \\ 0 & \ldots & 0 & v\end{array}\right)=y_{1} y_{2} \ldots y_{3 m}\left(\begin{array}{cccc}p_{1} & 0 & \ldots & 0 \\ 0 & \ddots & \ddots & \vdots \\ \vdots & \ddots & p_{n-1} & 0 \\ 0 & \ldots & 0 & v^{\prime}\end{array}\right)$.

These are done by repeatedly using the factorizations in (ii) of Theorem 1.4.

Case 1. $n=2^{m}$ for some integer $m$.

Since $\left[p_{1}\right] \leqq\left[p_{2}\right] \leqq \ldots \leqq\left[p_{n}\right]$, it is clear that

$$
p_{1} \oplus p_{2} \oplus \ldots \oplus p_{2^{p-1}} \sim q \leqq p_{m^{m-1}+1} \oplus \ldots \oplus p_{2^{m}} .
$$

It is obvious that $q \sim u\left(p_{1} \oplus p_{2} \oplus \ldots \oplus p_{2^{m-1}}\right) u^{*}$. It follows from Theorem 1.4 (ii) that there exist symmetries $z_{0}, z_{1}, z_{2}$ and a unitary $w_{1}$ in $p \mathscr{A} p$ such that $\left\|w_{1}-p\right\|<\frac{\varepsilon}{m}$ and

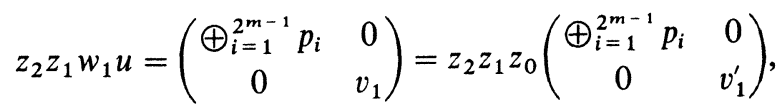

where the matrices are written with respect to the decomposition

$$
\left(\oplus_{i=1}^{2^{m-1}} p_{i}\right) \oplus\left(p-\oplus_{i=1}^{2^{m-1}} p_{i}\right)=p .
$$

Repeating this process recursively $m$ times, we reach the following factorizations:

$$
z_{2 m} z_{2 m-1} w_{m} z_{2 m-2} z_{2 m-3} w_{m-1} \ldots z_{2} z_{1} w_{1} u=\left(\begin{array}{cccc}
p_{1} & 0 & \ldots & 0 \\
0 & \ddots & \ddots & \vdots \\
\vdots & \ddots & p_{n-1} & 0 \\
0 & \ldots & 0 & v
\end{array}\right) \text { and }
$$




$$
y_{3 m} y_{3 m-1} y_{3 m-2} \ldots y_{3} y_{2} y_{1} u=\left(\begin{array}{cccc}
p_{1} & 0 & \ldots & 0 \\
0 & \ddots & \ddots & \vdots \\
\vdots & \ddots & p_{n-1} & 0 \\
0 & \ldots & 0 & v^{\prime}
\end{array}\right),
$$

where $z_{1}, z_{2}, \ldots, z_{2 m}, y_{1}, y_{2}, \ldots, y_{3 m}$ are some symmetries in $p \mathscr{A} p$ and $w_{1}, w_{2}, \ldots$, $w_{m}$ are some unitaries in $p \mathscr{A} p$ close to the identity $p$ in norm within $\frac{\varepsilon}{m}$. Set

$$
\begin{aligned}
& s_{1}^{\prime}=w_{1}^{*} z_{1} w_{1}, \\
& s_{2}^{\prime}=w_{1}^{*} z_{2} w_{1}, \\
& s_{3}^{\prime}=w_{1}^{*} w_{2}^{*} z_{3} w_{2} w_{1}, \\
& s_{4}^{\prime}=w_{1}^{*} w_{2}^{*} z_{4} w_{2} w_{1}, \\
& s_{5}^{\prime}=w_{1}^{*} w_{2}^{*} w_{3}^{*} z_{5} w_{3} w_{2} w_{1}, \ldots, \\
& s_{2 m}^{\prime}=w_{1}^{*} w_{2}^{*} \ldots w_{m}^{*} z_{2 m} w_{m} \ldots w_{2} w_{1}, \\
& u_{\varepsilon}=w_{1}^{*} w_{2}^{*} \ldots w_{m}^{*} .
\end{aligned}
$$

It is easy to check that $\left\|u_{\varepsilon}-p\right\|<\sum_{i=1}^{m} \frac{\varepsilon}{m}=\varepsilon$, and $\left\{s_{j}^{\prime}\right\}_{j=1}^{2 m}$ with $u_{\varepsilon}$ give the factorizations wanted.

Case 2. $n<2^{m}$, where $m$ is the smallest integer satisfying the inequality.

Set $q=\oplus_{i=1}^{n-2^{m-1}} p_{i}$. Then $q \sim q^{\prime} \leqq p-q$, since $0 \leqq n-2^{m-1}<2^{m-1}$. One can apply the same arguments as in the first step of the case 1 to $q$. The remaining job is the same as in the case 1 .

2.3. REMARK. Under the same assumptions as in Theorem 2.2 , one can obtain some upper bounds for the exponential rank and exponential length of $M_{n}(\mathscr{A})$. For example, if $p$ and $\left\{p_{i}\right\}$ are as in Theorem 2.2, then

$$
\begin{aligned}
& \operatorname{cer}(p \mathscr{A} p) \leqq\left\{\frac{m}{2}\right\}+\operatorname{cer}\left(p_{n} \mathscr{A} p_{n}\right)+\varepsilon \\
& \operatorname{cel}(p \mathscr{A} p) \leqq \frac{m}{2} \pi+\operatorname{cel}\left(p_{n} \mathscr{A} p_{n}\right),
\end{aligned}
$$

where $\{t\}$ is the smallest integer greater or equal to a number $t$.

After the original manuscript was written, $\mathrm{H}$. Lin proved a break-through result which asserted that

$$
\operatorname{cer}(\mathscr{A})<1+\varepsilon, \quad \text { and hence } \operatorname{cel}(\mathscr{A}) \leqq \pi
$$

for any $C^{*}$-algebra with $\operatorname{RR}(\mathscr{A})=0(\operatorname{tsr}(\mathscr{A})=1$ or not). Thus, the above esti- 
mates becomes worthless. We can not delete the above inequalities in this revision only because they are cited in some appeared or to be appeared articles of others.

2.4. EXAMPLE. The factorizations as in Theorem (2.2) are not always possible in an arbitrary $C^{*}$-algebra. For example, look at the commutative $C^{*}$-algebra $C\left(S^{3}\right)$ of all complex-valued continuous functions on the 3-sphere $S^{3}$. The homeomorphism $S^{3} \cong \mathrm{SU}(2)$ gives a unitary $u$ in $M_{2}\left(C\left(S^{3}\right)\right)$ which is not connected to the identity $I_{2}$, where $S U(2)$ is all unitary scalar matrices with determinant 1. Since each diagonal unitary of $M_{2}\left(C\left(S^{3}\right)\right)$ and all symmetries are in the identity path component of the unitary group of $M_{2}\left(C\left(S^{3}\right)\right)$, $u$ cannot be factored into a product of some symmetries and a diagonal unitary.

2.5. Corollary. Suppose that $\mathscr{A}$ is a $C^{*}$-algebra such that $\operatorname{RR}(\mathscr{A})=0$ and $\operatorname{tsr}(\mathscr{A})=1$, and suppose that $p$ is a full projection of $\mathscr{A}$ (i.e., the closed ideal generated by $p$ is $\mathscr{A}$ ) and $u$ is any unitary of $\mathscr{A}$.

(i) If $\mathscr{A}$ is unital, then there exist some symmetries $s_{1}, s_{2}, \ldots, s_{n}$ in $\mathscr{A}$ (for some $n \geqq 1$ ) such that

$$
u=s_{1} s_{2} \ldots s_{n}\left(\begin{array}{cc}
1-p & 0 \\
0 & u_{0}
\end{array}\right)
$$

where $u_{0}$ is a unitary in the hereditary $C^{*}$-subalgebra p $\mathscr{A} p$.

(ii) If $\mathscr{A}$ is non-unital, then there exist some symmetries $s_{1}, s_{2}, \ldots, s_{n}$ (for some $n \geqq 1$ ) and a unitary $u_{\varepsilon}$ in $\tilde{\mathscr{A}}$ such that

$$
\left\|u_{\varepsilon}-1\right\|<\varepsilon \text { and } u=s_{1} s_{2} \ldots s_{n} u_{\varepsilon}\left(\begin{array}{cc}
1-p & 0 \\
0 & u_{0}
\end{array}\right)
$$

where $u_{0}$ is a unitary in the hereditary $C^{*}$-subalgebra $p \mathscr{A} p$.

Proof. (i) Assume that $\mathscr{A}$ is unital. Since $p$ is a full projection, it was proved $[52,1.1]$ that

$$
1=q_{1} \oplus q_{2} \oplus \ldots \oplus q_{l} \text { and }\left[q_{1}\right] \leqq\left[q_{2}\right] \leqq \ldots \leqq\left[q_{l}\right] \leqq[p] .
$$

We may assume that $q_{l} \leqq p$. Then the conclusion follows from Theorem (2.2).

(ii) Assume that $\mathscr{A}$ is not unital. Since $\operatorname{RR}(\mathscr{A})=0$, there is an approximate identity of $\mathscr{A}$ consisting of projections. Then we can choose a projection $q \in \mathscr{A}$ and a unitary $u_{1 \varepsilon}$ in $\tilde{\mathscr{A}}$ such that

$$
\left\|u_{1 \varepsilon}-1\right\|<\frac{\varepsilon}{2}, \quad q>p, \quad \text { and } \quad u=u_{1 \varepsilon}\left(\begin{array}{cc}
1-q & 0 \\
0 & u_{1}
\end{array}\right)
$$

where $u_{1}$ is a unitary in $q \mathscr{A} q$ (see the proof of $[50,4.1]$ or $[54,2.6]$, for example). Now the problem has been reduced to the unital $C^{*}$-algebra $q \mathscr{A} q$. 


\section{Factorizations in $U(M())$ ).}

We now consider the same type of factorizations in the unitary group of the multiplier algebra $M(\mathscr{A})$ in case $\mathscr{A}$ is non-unital. G. A. Elliott proved [22,2.1] that every unitary algebra of a matroid algebra can be approximated in norm by the product of two block diagonal unitaries (with respect to two different decompositions of the identity). The same technique also applies to a $C^{*}$-algebra with $\operatorname{RR}(\mathscr{A})=0$ and $\operatorname{tsr}(\mathscr{A})=1$.

In this section we provide two different types of factorizations in the unitary group of $M(\mathscr{A})$; one is Proposition 3.1 which is proved by the combination of Elliott's technique in [22,2.1] and Theorem 1.4; the other is Theorem 3.2 which is proved by the combination of the technique of [22], [59,1.1] and Theorem 1.4.

3.1. Proposition. Assume that $\varepsilon$ is any number, and $\mathscr{A}$ is a non-unital, $\sigma$-unital $C^{*}$-algebra such that $\operatorname{R} \operatorname{R}(\mathscr{A})=0$ and $\operatorname{tsr}(\mathscr{A})=1$. Then every unitary $u$ of $M(\mathscr{A})$ can be factored as $u=u_{\varepsilon} s u_{0}$, where $\left\|u_{\varepsilon}-1\right\|<\varepsilon$, s is a symmetry, $u_{0}$ is a diagonal unitary $\oplus_{i=1}^{\infty} u_{i}^{\prime}$, where $u_{i}^{\prime}$ is a unitary of the corner $\left(e_{n_{i}}-e_{n_{i-1}}\right) \mathscr{A}\left(e_{n_{i}}-e_{n_{i-1}}\right)$ $(\forall i \geqq 1)$, and $\left\{e_{n}\right\}$ is an approximate identity of $\mathscr{A}$ consisting of a sequence of increasing projections.

Proof. Using the technique of G. A. Elliott in [22,2.1], one can choose an approximate identity of $\mathscr{A}$ consisting of an increasing sequence of projections, say $\left\{e_{n}\right\}$. For any positive number $\varepsilon$ there exists a unitary $v$ in $M(\mathscr{A})$ with the following properties:

$$
\|v-u\|<\varepsilon / 2 \quad \text { and } \quad v=\left(\sum_{i=1}^{\infty} \oplus u_{i}\right)\left(\sum_{i=1}^{\infty} \oplus v_{i}\right),
$$

where

$$
\begin{aligned}
& u_{i} \in U\left(\left(e_{2 i-1}-e_{2 i-1}\right) \mathscr{A}\left(e_{2 i+1}-e_{2 i-1}\right)\right), \\
& v_{i} \in U\left(\left(e_{2 i}-e_{2 i-2}\right) \mathscr{A}\left(e_{2 i}-e_{2 i-2}\right) \quad\left(i \geqq 1, e_{0}=0\right) .\right.
\end{aligned}
$$

Here one may need to change the notations after choosing a subsequence of $\left\{e_{n}\right\}$. Applying Theorem 1.4 to each $u_{i}$, one can write

$$
u_{i}=s_{i} w_{i}\left(\begin{array}{cc}
u_{1 i} & 0 \\
0 & u_{2 i}
\end{array}\right) \quad(i \geqq 1)
$$

where the matrix decomposition is with respect to

$$
\left(e_{2 i+1}-e_{2 i}\right) \oplus\left(e_{2 i}-e_{2 i-1}\right)=e_{2 i+1}-e_{2 i-1},
$$

$s_{i}$ is symmetry, $w_{i}$ is a unitary of the corner $U\left(\left(e_{2 i+1}-e_{2 i-1}\right) \mathscr{A}\right.$ $\left.\left(e_{2 i+1}-e_{2 i-1}\right)\right)$ such that 


$$
\left\|w_{i}-\left(e_{2 i+1}-e_{2 i-1}\right)\right\|<\varepsilon / 2 \quad(i \geqq 1) .
$$

Set $s^{\prime}=\sum_{i=1}^{\infty} \oplus s_{i}$, and $w=\sum_{i=1}^{\infty} \oplus w_{i}$. Then $\|w-1\|<\varepsilon / 2$ and

$$
\sum_{i=1}^{\infty} \oplus u_{i}=s^{\prime} w \sum_{i=1}^{\infty}\left(u_{1 i} \oplus u_{2 i}\right)
$$

Clearly, $\sum_{i=1}^{\infty}\left(u_{1 i} \oplus u_{2 i}\right) \sum_{i=1}^{\infty} \oplus v_{i}:=u_{0}$ is a diagonal unitary with respect to the decomposition $\sum_{i=1}^{\infty}\left(e_{2 i}-e_{2 i-2}\right)=1$. Thus

$$
u=\left(u v^{*}\right) v=\left(u v^{*}\right) s w u_{0}=\left(u v^{*} w\right)\left(w^{*} s^{\prime} w\right) u_{0}
$$

has the desired form, since

$$
\left\|u v^{*} w-1\right\|=\left\|u-w^{*} v\right\| \leqq\|u-v\|+\left\|w^{*}-1\right\|<\varepsilon .
$$

Set $u_{\varepsilon}=u v^{*} w$ and $s=w^{*} s^{\prime} w$ as wanted.

3.2. In case $\mathscr{A}$ is a $\sigma$-unital, non-unital, simple $C^{*}$-algebra of real rank zero, we proved $[59,1.1]$ that every projection $p$ in $M(\mathscr{A})$ not in $\mathscr{A}$ can be written as

$$
p=p_{1} \oplus p_{2} \oplus \ldots \oplus p_{2^{n}},
$$

where $n \geqq 1$ can be any integer and $\left\{p_{i}\right\}_{i=1}^{2 n}$ are mutually equivalent orthogonal projections of $M(\mathscr{A})$ (of course not in $\mathscr{A}$ ). In particular, the identity of $M(\mathscr{A})$ can be written as

$$
1=p_{1} \oplus p_{2} \oplus \ldots \oplus p_{2^{n}}
$$

Guaranteed by $[56,1.2]$, we can write each $p_{i}$ into a direct sum of a sequence of projections of $\mathscr{A}$, say

$$
p_{i}=f_{i 1} \oplus f_{i 2} \oplus \ldots \oplus f_{i j} \oplus \ldots
$$

Furthermore, we can assume that

$$
f_{1 j} \sim f_{2 j} \sim \ldots \sim f_{2^{n} j} \text { for all } j \geqq 1 .
$$

Now we have the following factorization of unitaries in $M(\mathscr{A})$ :

3.3. THEOREM. Suppose that $\mathscr{A}$ is a $\sigma$-unital, non-unital, simple $C^{*}$-algebra with $\operatorname{RR}(\mathscr{A})=0$ and $\operatorname{tsr}(\mathscr{A})=1$. Write $1=p_{1} \oplus p_{2} \oplus \ldots \oplus p_{2^{n}}$ for some $n \geqq 2$, where $p_{i} \sim p_{j}\left(\forall 1 \leqq i, j \leqq 2^{n}\right)$. If $u$ is any unitary in $M(\mathscr{A})$ and $\varepsilon$ is any positive number, then there exist a unitary $u_{\varepsilon}$ in $M(\mathscr{A})$ with $\left\|u_{\varepsilon}-1\right\|<\varepsilon$ and $2 n$ symmetries $\left\{s_{k}\right\}_{k=0}^{2 n}$ in $M(\mathscr{A})$ such that

$$
u=u_{\varepsilon} s_{0} s_{1} s_{2} \ldots s_{2 n}\left(\begin{array}{cccc}
p_{1} & 0 & \ldots & 0 \\
0 & \ddots & \ddots & \vdots \\
\vdots & \ddots & p_{2^{n}-1} & 0 \\
0 & \ldots & 0 & v
\end{array}\right)
$$


where $v$ is a unitary of the corner $p_{2^{n}} M(\mathscr{A}) p_{2^{n}}$ whose matrix form with respect to the decomposition $p_{2^{n}}=f_{2^{n_{1}}} \oplus f_{2^{n_{2}}} \oplus \ldots \oplus f_{2^{n_{j}}} \oplus \ldots$ is block-diagonal; i.e., $v=$ $\sum_{i=1}^{\infty} \oplus v_{i}$, each $v_{i}$ is a unitary in the corner $\left.\left(f_{2^{n} m_{i}}-f_{2^{n} m_{i-1}}\right) \mathscr{A}\left(f_{2^{n} m_{i}}-f_{2^{n} m_{i-1}}\right)\right)$ for some sequence $\left\{m_{i}\right\}$.

Proof. Set $e_{m}=\sum_{j=1}^{m}\left(f_{1 j} \oplus f_{2 j} \oplus \ldots \oplus f_{2^{n} j}\right)$ for all $m \geqq 1$. Then $\left\{e_{m}\right\}$ is an increasing approximate identity of $\mathscr{A}$ consisting of projections. It follows from Proposition 3.1 that there exist a unitary $w_{0}$ with $\left\|w_{0}-1\right\|<\frac{\varepsilon}{2}$ and a symmetry $s_{0}^{\prime}$ such that

$$
s_{0}^{\prime} w_{0} u=\sum_{i=1}^{\infty} \oplus u_{i},
$$

where $u_{i}$ is a unitary of $\left(e_{m_{i}}-e_{m_{i-1}}\right) \mathscr{A}\left(e_{m_{i}}-e_{m_{i-1}}\right)$ for all $i \geqq 1\left(e_{m_{0}}=0\right)$. Clearly,

$$
\begin{gathered}
e_{m_{i}}-e_{m_{i-1}}=\sum_{j=m_{i-1}+1}^{m_{i}}\left(f_{1 j} \oplus f_{2 j} \oplus \ldots \oplus f_{2^{n_{j}}}\right) \\
=\left(\sum_{j=m_{i-1}+1}^{m_{i}} f_{1 j}\right) \oplus\left(\sum_{j=m_{i-1}+1}^{m_{i}} f_{2 j}\right) \oplus \ldots \oplus\left(\sum_{j=m_{i-1}+1}^{m_{i}} f_{2^{n_{j}}}\right), \quad \text { and } \\
\sum_{j=m_{i-1}+1}^{m_{i}} f_{1 j} \sim\left(\sum_{j=m_{i-1}+1}^{m_{i}} f_{2 j}\right) \sim \ldots \sim \sum_{j=m_{i-1}+1}^{m_{i}} f_{2^{n_{j}}} .
\end{gathered}
$$

It then follows from Theorem 2.2 that there are a unitary $u_{i}^{\prime}$ and $2 n$ symmetries in $\left.\left(e_{m_{i}}-e_{m_{i-1}}\right) \mathscr{A} e_{m_{i}}-e_{m_{i-1}}\right)$, say $s_{i 1}, s_{i 2}, \ldots, s_{i, 2 n}$ (notice that $n$ is the smallest integer such that $\left.m \leqq 2^{n}\right)$, such that $\left\|u_{i}^{\prime}-\left(e_{m_{i}}-e_{m_{i-1}}\right)\right\|<\frac{\varepsilon}{2}$ and

$$
u_{i}=u_{i}^{\prime} s_{i 1} s_{i 2} \ldots s_{i, 2 n}\left(\begin{array}{cccc}
\sum_{j=m_{i-1}+1}^{m_{i}} f_{1 j} & 0 & \ldots & 0 \\
0 & \ddots & \ddots & \vdots \\
\vdots & \ddots & \sum_{j=m_{i-1}+1}^{m_{i}} f_{2^{n-1, j}} & 0 \\
0 & \ldots & 0 & v_{i}
\end{array}\right) \text {, }
$$

where $v_{i}$ is a unitary in $\left(\sum_{j=m_{i-1}+1}^{m_{i}} \oplus f_{2^{n_{j}}}\right) \mathscr{A}\left(\sum_{j=m_{i-1}+1}^{m_{i}} \oplus f_{2^{n_{j}}}\right)$.

Set $v=\sum_{i=1}^{\infty} \oplus v_{i}$. Then $v_{i}$ is a unitary of the corner $\sum_{j=m_{i-1}+1}^{m_{i}}\left(f_{2^{n_{j}}}-\right.$ $\left.\left.f_{2^{n}, j-1}\right) \mathscr{A} \sum_{j=m_{i-1}+1}^{m_{i}}\left(f_{2^{n_{j}}}-f_{2^{n, j-1}}\right)\right)$ for each $i \geqq 1$. Thus, $v$ is a block-diagonal unitary of $p_{2^{n}} \mathscr{A} p_{2^{n}}$. Set $s_{j}=\sum_{i=1}^{\infty} \oplus s_{i j}$ for $1 \leqq j \leqq 2 n$ and $w_{1}=\sum_{i=1}^{\infty} \oplus u_{i}^{\prime}$. It is a routine to check that $s_{j}$ 's are symmetries in $M(\mathscr{A})$ and $w_{1}$ is a unitary of $M(\mathscr{A})$ with $\left\|w_{1}-1\right\|<\frac{\varepsilon}{2}$. Now one can easily check that 


$$
u=\left(w_{0}^{*} w_{1}\right)\left(w_{1}^{*} s_{0}^{\prime} w_{1}\right) s_{1} s_{2} \ldots s_{2 n}\left(\begin{array}{cccc}
p_{1} & 0 & \ldots & 0 \\
0 & \ddots & \ddots & \vdots \\
\vdots & \ddots & p_{2^{n}-1} & 0 \\
0 & \ldots & 0 & v
\end{array}\right)
$$

Set $u_{\varepsilon}=w_{0}^{*} w_{1}$ and $s_{0}=w_{1}^{*} s_{0}^{\prime} w_{1}$, as desired.

\section{4. $\operatorname{tsr}(\mathscr{A})$ and factorizations in $\mathscr{A} \otimes \mathscr{K}$.}

\section{1. $U(\tilde{\mathscr{A}})$ and $\mathscr{P}(\mathscr{A})$.}

Let $\mathscr{A}$ be a $C^{*}$-algebra (may not be of real rank zero). A natural action of the unitary group $U(\tilde{\mathscr{A}})$ on the Grassmann space $\mathscr{P}(\mathscr{A})$ is defined by

$$
(u, p) \mapsto u p u^{*} \quad \forall u \in U(\tilde{\mathscr{A}}) \text { and } \forall p \in \mathscr{P}(\mathscr{A}) \text {. }
$$

If $p$ is a fixed nontrivial projection, the orbit of $p$ under this action, denoted by

$$
\mathscr{G}_{p}:=\left\{u p u^{*}: u \in U(\tilde{\mathscr{A}})\right\},
$$

is the union of those path components of $\mathscr{P}(\mathscr{A})$ consisting of projections unitarily equivalent to $p$. If $U(M(\mathscr{A}))$ is connected, then of course $\mathscr{G}_{p}$ is connected for any $p \in \mathscr{P}(\mathscr{A})$. On the other hand, $\mathscr{G}_{p}$ still can be connected even in case $U(M(\mathscr{A}))$ is not connected. For example, it was proved ([21, 2.4], [48, 3.4], [51]) that $\mathscr{G}_{p}$ consists of only one path component in cases either $\mathscr{A}$ is purely infinite and simple or $\mathscr{A}$ satisfies $\operatorname{RR}(\mathscr{A})=0$ and $\operatorname{tsr}(\mathscr{A})=1$ (not necessarily simple), no matter $U(M(\mathscr{A}))$ is connected or not. It is conceivable that $\mathscr{G}_{p}$ is connected for many other $C^{*}$-algebras. How the internal natures of a $C^{*}$-algebra relate to the connectedness of $\mathscr{G}_{p}$ is almost a total mystery. Our interest in this section is the connection between $\mathscr{G}_{p}$ is almost a total mystery. Our interest in this section is the connection between $\mathscr{G}_{p}$ and $U(\tilde{\mathscr{A}})$.

From now on, for any $n \geqq 1$ the unitary group of $M_{n}(\tilde{\mathscr{A}})$ is denoted by $U_{n}(\mathscr{A})$ and the identity path component of $U_{n}(\mathscr{A})$ is denoted by $U_{n}^{0}(\mathscr{A})$. The unitary group of the unitization of $\mathscr{A} \otimes \mathscr{K}$ is denoted by $U_{\infty}(\mathscr{A})$.

If $m<n, U_{m}(\mathscr{A})$ is naturally embedded into $U_{n}(\mathscr{A})$ by

$$
i_{m, n}: u_{m} \mapsto\left(\begin{array}{cc}
u_{m} & 0 \\
0 & I_{n-m}
\end{array}\right) \text {. }
$$

Of course, $U_{m}^{0}(\mathscr{A})$ is correspondingly embedded in $U_{n}^{0}(\mathscr{A})$. Clearly, $i_{m . n}$ induces a group homomorphism:

$$
\phi_{m, n}: U_{m}(\mathscr{A}) / U_{m}^{0}(\mathscr{A}) \rightarrow U_{n}(\mathscr{A}) / U_{n}^{0}(\mathscr{A}) .
$$

However, the map $\phi_{m . n}$ is neither injective nor surjective in general $([3,8.1 .2])$. Similarly, the natural embedding of $U_{n}(\mathscr{A})$ into $U_{\infty}(\mathscr{A})$ defined by 


$$
i_{n, \infty}: \mapsto\left(\begin{array}{cc}
u_{n} & 0 \\
0 & 1-I_{n}
\end{array}\right)
$$

induces a group homomorphism

$$
\phi_{n, \infty}: U_{n}(\mathscr{A}) / U_{n}^{0}(\mathscr{A}) \rightarrow U_{\infty}(\mathscr{A}) / U_{\infty}^{0}(\mathscr{A}):=K_{1}(\mathscr{A}) ;
$$

The homomorphism $\phi_{n, \infty}$ is again neither injective nor surjective in general. On the other hand, there are many interesting $C^{*}$-algebras such that $\phi_{n, \infty}$ is an isomorphism for an integer $n$. For example, M. A. Rieffel $[40,2.10]$ proved that if $\mathscr{A}$ is a unital $C^{*}$-algebra such that $\operatorname{tsr}(\mathscr{A})<\infty$, then $\phi_{n, \infty}$ is an isomorphism whenever $n \geqq \operatorname{tsr}(\mathscr{A})$; J. Cuntz proved [17] that if $\mathscr{A}$ is a purely infinite, simple, unital $C^{*}$-algebra, then $\phi_{1, \infty}$ is an isomorphism (it holds also in case $\mathscr{A}$ is non-unital $[50, \S 4])$; but $\operatorname{tsr}(\mathscr{A})=+\infty$. This example shows that a finite topological stable rank is not a necessary condition for the occurrence of an isomorphism $\phi_{n, \infty}$ for an integer $n$.

For any projection $p \in \mathscr{P}(\mathscr{A})$, we will consider the relation of the connectedness of $\mathscr{G}_{p}$ and the factorization in the unitary group $U(\mathscr{A})$, and we will then clarify when $\phi_{n, \infty}$ is surjective for an integer $n$.

4.2. Lemma. Assume that $\mathscr{A}$ is any $C^{*}$-algebra and $\left.p \in \mathscr{P}(\mathscr{A})\right)$. Then the following are equivalent:

(i) $\mathscr{G}_{p}$ is connected.

(ii) Every unitary $u$ of $\tilde{\mathscr{A}}$ is homotopic (in $U(\tilde{\mathscr{A}})$ ) to a unitary with the form

$$
\left(\begin{array}{cc}
u_{1} & 0 \\
0 & u_{2}
\end{array}\right)
$$

where $u_{1}$ is in $U(p \mathscr{A} p)$ and $u_{2}$ is in $U((1-p) \tilde{\mathscr{A}}(1-p))$.

(iii) Every unitary $u$ of $\tilde{\mathscr{A}}$ can be written as

$$
u=s_{1} s_{2} \ldots s_{l}\left(\begin{array}{cc}
u_{1} & 0 \\
0 & u_{2}
\end{array}\right)
$$

where $\left\{s_{i}\right\}_{i=1}^{l}$ is a set of symmetries in $U(\tilde{\mathscr{A}}), u_{1} \in U(p \mathscr{A} p)$ and $u_{2} \in U((1-p)$ $\tilde{\mathscr{A}}(1-p))$.

Proof. If $\{u(t): t \in[0,1]\}$ is a path in $U(\tilde{\mathscr{A}})$, then $\left\{u(t) p u(t)^{*}: t \in[0,1]\right\}$ is a path in $\mathscr{P}(\mathscr{A})$. Hence, it is trivial that (ii) implies (i) and (iii) implies (i). Since each isometry is in the identity component of $U(\tilde{\mathscr{A}})$, then it is clear that (iii) implies (ii), We need only to show that (i) implies (iii).

By assumption $u p u^{*}$ is homotopic to $p$. One can choose a finite number of projections $p_{1}, p_{2}, \ldots, p_{l}$ such that $p_{l}=p$ and $\left\|u p u^{*}-p_{1}\right\|<1$, and $\left\|p_{i}-p_{i+1}\right\|<1$ for $1 \leqq i \leqq l-1$. Set 


$$
\begin{aligned}
& s_{1}=\left(1-\left(u p u^{*}-p_{1}\right)^{2}\right)^{-\frac{1}{2}}\left(u p u^{*}+p_{1}-1\right), \quad \text { and } \\
& s_{i+1}=\left(1-\left(p_{i}-p_{i+1}\right)^{2}\right)^{-\frac{1}{2}}\left(p_{i}+p_{i+1}-1\right) \quad \forall 1 \leqq i \leqq l-1 .
\end{aligned}
$$

Then $s_{1}, s_{2}, \ldots, s_{l}$ are symmetries such that $s_{1} u p u^{*} s_{1}=p_{1}$ and $s_{i} p_{i} s_{i}=p_{i+1}$ for $1 \leqq i \leqq l-1$. It follows that

$$
s_{l} \ldots s_{2} s_{1} u p u^{*} s_{1} s_{2} \ldots s_{l}=p,
$$

and hence

$$
u=s_{1} s_{2} \ldots s_{l}\left(\begin{array}{cc}
u_{1} & 0 \\
0 & u_{2}
\end{array}\right)
$$

where $u_{1}$ is a unitary of $p \mathscr{A} p$ and $u_{2}$ is a unitary of $(1-p) \tilde{\mathscr{A}}(1-p)$, as wanted.

4.3. TheOREM. Assume that $\mathscr{A}$ is any $C^{*}$-algebra and $n>m \geqq n-m$. Let $p_{m}=\operatorname{diag}(1,1, \ldots, 1,0, \ldots, 0)$, an $n \times n$ diagonal matrix in $M_{n}(\tilde{\mathscr{A}})$. Then the following hold:

(i) The orbit $\mathscr{G}_{p_{m}}$ of $p_{m}$ under the action of $U_{n}(\mathscr{A})$ is connected subset of $\mathscr{P}\left(M_{n}(\tilde{\mathscr{A}})\right)$ if and only if $\phi_{m, n}: U_{m}(\mathscr{A}) \rightarrow U_{n}(\mathscr{A}) / U_{n}^{0}(\mathscr{A})$ is surjective.

(ii) If $\phi_{m, n}$ is surjective, then every unitary $u_{n}$ of $U_{n}(\mathscr{A})$ can be written as

$$
u_{n}=s_{1} s_{2} \ldots s_{l}\left(\begin{array}{cc}
u_{m}^{\prime} & 0 \\
0 & I_{n-m}
\end{array}\right)
$$

for some $l \geqq 1$ and symmetries $s_{1}, s_{2}, \ldots, s_{l}$ of $M_{n}(\tilde{\mathscr{A}})$, where $u_{m}^{\prime}$ is a unitary of $M_{m}(\tilde{\mathscr{A}})$.

REMARK. The condition $n-m \leqq m$ is not essential, because either $n-m \leqq m$ or $m \leqq n-m$ holds and that $\mathscr{G}_{p_{m}}$ is a connected subset of $\mathscr{P}\left(M_{n}(\tilde{\mathscr{A}})\right)$ iff $\mathscr{G}_{p_{n-m}}$ is.

Proof. (i) Assume that $\phi_{m, n}$ is surjective. Then every unitary $u_{n}$ of $U_{n}(\mathscr{A})$ is homotopic to a unitary $v$ with the following form

$$
\left(\begin{array}{cc}
u_{m} & 0 \\
0 & I_{n-m}
\end{array}\right) \text {. }
$$

It follows that $u_{n} p_{m} u_{n}^{*}$ is homotopic to $v p_{m} v^{*}=p_{m}$. Thus, the orbit of $p_{m}$ is connected as a subset of $\mathscr{P}\left(M_{n}(\tilde{\mathscr{A}})\right)$.

Conversely, if the orbit $\mathscr{G}_{p_{m}}$ is a connected subset of $\mathscr{P}\left(M_{n}(\mathscr{A})\right)$, and if $u_{n}$ is any unitary of $M_{n}(\mathscr{A})$, then $u_{n} p_{m} u_{n}^{*}$ is homotopic to $p_{m}$. It follows from Lemma (4.2) that $u_{n}$ is homotopic to a unitary $w$ with the form

$$
\left(\begin{array}{cc}
u_{m} & 0 \\
0 & u_{n-m}
\end{array}\right)
$$


where $u_{m}$ is a unitary of $M_{m}(\mathscr{A})$ and $u_{n-m}$ is a unitary of $M_{n-m}(\mathscr{A})$. If $n-m \leqq m$, there is a partial isometry $v$ of $M_{n}(\mathscr{A})$ such that

$$
v v^{*}=I_{n-m} \quad \text { and } \quad v^{*} v=\sum_{i=1}^{n-m} 1 \otimes e_{i i}
$$

Then

$$
\left(\begin{array}{cc}
u_{m} & 0 \\
0 & u_{n-m}
\end{array}\right)
$$

can be written as the product $w_{1} w_{2}$, where

$$
\begin{gathered}
w_{1}:=\left(\begin{array}{ccc}
\left(v^{*} u_{n-m}^{*} v\right) & 0 & 0 \\
0 & \left(\sum_{i=n-m+1}^{m} 1 \otimes e_{i i}\right) & 0 \\
0 & 0 & u_{n-m}
\end{array}\right) \text { and } \\
w_{2}:=\left(\begin{array}{ccc}
\left(v^{*} u_{n-m} v \oplus \sum_{i=n-m+1}^{m} 1 \otimes e_{i i}\right) u_{m} & 0 \\
0 & I_{n-m}
\end{array}\right) .
\end{gathered}
$$

On the other hand, $w_{1}$ can be written as a product of two symmetries as follows:

$$
w_{1}=\left(\begin{array}{ccc}
0 & 0 & v^{*} \\
0 & \sum_{i=n-m+1}^{m} 1 \otimes e_{i i} & 0 \\
v & 0 & 0
\end{array}\right)\left(\begin{array}{ccc}
0 & 0 & v^{*} u_{n-m} \\
0 & \sum_{i=n-m+1}^{m} 1 \otimes e_{i i} & 0 \\
u_{n-m}^{*} v & 0 & 0
\end{array}\right) .
$$

Set $u_{m}^{\prime}=\left(v^{*} u_{n-m} v \oplus \sum_{i=n-m+1}^{m} 1 \otimes e_{i i}\right) u_{m}$. Then $w_{1} w_{2}$ is homotopic to a unitary with the form

$$
\left(\begin{array}{cc}
u_{m}^{\prime} & 0 \\
0 & I_{n-m}
\end{array}\right)
$$

which is the image of an element in $U_{m}(\mathscr{A})$ under $\phi_{m, n}$. We have proved the conclusion (i).

(ii) If $\phi_{m, n}$ is surjective, then $\mathscr{G}_{p_{m}}$ is a connected subset of $\mathscr{P}\left(M_{n}(\tilde{\mathscr{A}})\right)$. It follows from Lemma 4.2 that

$$
u_{n}=s_{1} s_{2} \ldots s_{1-2}\left(\begin{array}{cc}
v_{m} & 0 \\
0 & u_{n-m}
\end{array}\right)
$$

for a finite set of symmetries $\left\{s_{j}\right\}_{j=1}^{l-2}$ in $U_{n}(\mathscr{A})$. As in the last paragraph, we can rewrite

$$
\left(\begin{array}{cc}
v_{m} & 0 \\
0 & u_{n-m}
\end{array}\right)
$$

as a product of two symmetries and a unitary of the form 


$$
\left(\begin{array}{cc}
u_{m}^{\prime} & 0 \\
0 & I_{n-m}
\end{array}\right) .
$$

We then have the desired factorization.

The following corollary is immediate from Theorem 4.3 (just let $m=n-1$ ):

4.4. Corollary. Let $\mathscr{A}$ be any unital $C^{*}$-algebra and $n \geqq 2$. Then

$$
\phi_{n-1, n}: U_{n-1}(\mathscr{A}) / U_{n-1}^{0}(\mathscr{A}) \rightarrow U_{n}(\mathscr{A}) / U_{n}^{0}(\mathscr{A})
$$

is surjective if and only if $\mathscr{G}_{n}(\mathscr{A})$ is a connected subset of $\mathscr{P}\left(M_{n}(\tilde{\mathscr{A}})\right)$; where $\mathscr{G}_{n}(\mathscr{A})$ is the orbit, under the action of $U_{n}(\mathscr{A})$, of the projection

$$
e_{n}=\left(\begin{array}{ccc}
0 & \ldots & \ldots \\
\vdots & \ddots & \ddots \\
\vdots & \ddots & 0 \\
0 & \ldots & 1
\end{array}\right)_{n \times n}
$$

4.5. COROLlaRY. Let $m \geqq 1$ be a fixed integer. Then the following implications hold: (i) $\Rightarrow$ (ii) $\Rightarrow$ (iii) $\Rightarrow$ (iv):

(i) $\mathscr{G}_{n}(\mathscr{A})$ is connected subset of $\mathscr{P}\left(M_{n}(\mathscr{A})\right)$ for all $n>m$.

(ii) For any $n>m$, every unitary $u_{n} \in U_{n}(\mathscr{A})$ can be written as a product of the following form

$$
u=s_{1} s_{2} \ldots s_{l}\left(\begin{array}{cc}
u_{m} & 0 \\
0 & I_{n-m}
\end{array}\right),
$$

where $\left\{s_{j}\right\}_{j=1}^{l}$ is a set of symmetries in $U_{n}(\mathscr{A})$ and $u_{m} \in U_{m}(\mathscr{A})$.

(iii) Every unitary $u$ of $U_{\infty}(\mathscr{A})$ can be factored as the following form:

$$
u=u_{\varepsilon} s_{1} s_{2} \ldots s_{l}\left(\begin{array}{cc}
u_{m} & 0 \\
0 & \lambda\left(\sum_{i=m+1}^{\infty} 1 \otimes e_{i i}\right)
\end{array}\right),
$$

where $\lambda$ is a complex number with $|\lambda|=1,\left\{s_{j}\right\}_{j=1}^{l}$ is a set of symmetries in $U_{\infty}(\mathscr{A})$ such that $s_{j}\left(1-\sum_{i=1}^{n_{0}} 1 \otimes e_{i i}\right)=1-\sum_{i=1}^{n_{0}} 1 \otimes e_{i i}$ for some fixed $n_{0}, u_{\varepsilon}$ is a unitary in $U_{\infty}(\mathscr{A})$ close to the identity in norm within any given positive number $\varepsilon$, and $u_{m}$ is a unitary in $U_{m}(\mathscr{A})$.

(iv) The map $\phi_{m, \infty}: U_{m}(\mathscr{A}) / U_{m}^{0}(\mathscr{A}) \rightarrow K_{1}(\mathscr{A})$ is a surjective homomorphism (and hence $K_{1}(\mathscr{A})$ is a quotient group of $\left.U_{m}(\mathscr{A}) / U_{m}^{0}(\mathscr{A})\right)$.

Proof. Assume that $\mathscr{G}_{n}(\mathscr{A})$ is a connected subset of $\mathscr{P}\left(M_{n}(\mathscr{A})\right)$. By Theorem 4.3 there are symmetries $s_{1}, s_{2}, \ldots, s_{l_{1}}$ such that

$$
u=s_{1} s_{2} \ldots s_{l_{1}}\left(\begin{array}{cc}
u_{n-1} & 0 \\
0 & 1-I_{n-1}
\end{array}\right)
$$


where $u_{n-1}$ is a unitary of $U_{n-1}(\mathscr{A})$. Repeating this argument $n-m$ times recursively, one sees that (i) implies (ii).

Assume that (ii) holds. Let $u_{\infty}$ be any unitary of $U_{\infty}(\mathscr{A})$. For any positive number $\varepsilon$, there exist an integer $n_{0} \geqq 1$, a unitary $u_{n_{0}} \in U_{n_{0}}(\mathscr{A})$, and a complex number $\lambda$ with $|\lambda|=1$ such that

$$
\left(\begin{array}{cc}
u_{n_{0}} & 0 \\
0 & \lambda\left(\sum_{i=n_{0}+1}^{\infty} 1 \otimes e_{i i}\right)
\end{array}\right)
$$

is close to $u_{\infty}$ in norm within $\varepsilon$. Then one can write

$$
u_{\infty}=u_{\varepsilon}\left(\begin{array}{cc}
u_{n_{0}} & 0 \\
0 & \lambda\left(\sum_{i=n_{0}+1}^{\infty} 1 \otimes e_{i i}\right)
\end{array}\right),
$$

where

$$
u_{\varepsilon}=u_{\infty}\left(\begin{array}{cc}
u_{n_{0}}^{*} & 0 \\
0 & \lambda\left(\sum_{i=n_{0}+1}^{\infty} 1 \otimes e_{i i}\right)
\end{array}\right)
$$

such that $\left\|u_{\varepsilon}-1\right\|<\varepsilon$. Applying (ii) to $u_{n_{0}}$, the conclusion (iii) follows.

It is obvious that (iii) implies (iv).

4.6. Corollary. If $\operatorname{tsr}(\mathscr{A})<+\infty$ (in particular, if $\mathscr{A}=C(X)$ for some compact Hausdorff space $X$ whose covering dimension $\operatorname{dim}(X)<+\infty)$, then for any $n \geqq \operatorname{tsr}(\mathscr{A})$

(1) $\mathscr{G}_{n+1}(\mathscr{A})$ is a connected subset of $\mathscr{P}\left(M_{n}(\tilde{\mathscr{A}})\right)$;

(2) every unitary $u_{\infty}$ of $U_{\infty}(\mathscr{A})$ can be written as a product of the following form

$$
u_{\infty}=u_{\varepsilon} s_{1} s_{2} \ldots s_{l}\left(\begin{array}{cc}
u_{n} & 0 \\
0 & \lambda\left(\sum_{i=m+1}^{\infty} 1 \otimes e_{i i}\right)
\end{array}\right),
$$

where $s_{1}, \ldots, s_{1}, u_{n}$, and $u_{\varepsilon}$ are as explained in Corollary 4.5 (iii); and

(3) $\tilde{\mathscr{A}} \otimes \mathscr{K}$ is generated (as a $C^{*}$-algebra) by unitaries in $U_{n}(\mathscr{A})$ and projections.

PROOF. If $\operatorname{tsr}(\mathscr{A})<+\infty$, then $\phi_{n, n+1}$ is surjective for any $n \leqq \operatorname{tsr}(\mathscr{A})[40$, 2.10]). Hence, the conclusions (1) and (2) follows immediately from Corollary 4.4 and Corollary 4.5. Since each symmetry is of the form $2 r-1$ for some projections in $\tilde{\mathscr{A}} \otimes \mathscr{K}$, the conclusion (3) follows from the factorization in (2).

4.7. CoROllary. If $\mathscr{G}_{m}(\mathscr{A})$ is connected for all $m=2,3, \ldots, n$, then every unitary $u$ of $M_{n}(\mathscr{A})$ can be written as

$$
u=s_{1} s_{2} \ldots s_{l}\left(\begin{array}{cccc}
u_{1} & 0 & \ldots & 0 \\
0 & u_{2} & \ddots & \vdots \\
\vdots & \ddots & \ddots & 0 \\
0 & \ldots & 0 & u_{n}
\end{array}\right)=s_{1}^{\prime} s_{2}^{\prime} \ldots s_{k}^{\prime}\left(\begin{array}{cccc}
1 & 0 & \ldots & 0 \\
0 & \ddots & \ddots & \vdots \\
\vdots & \ddots & 1 & 0 \\
0 & \ldots & 0 & v
\end{array}\right),
$$


where $\left\{s_{j}, s_{i}^{\prime}\right\}$ is a finite set of symmetries of $M_{n}(\tilde{\mathscr{A}})$ and $\left\{v, u_{i}\right\} \subset U(\mathscr{A})$. Consequently, $U_{1}(\mathscr{A}) / U^{0}(\mathscr{A}) \stackrel{\phi_{1, \infty}}{\longrightarrow} K_{1}(\mathscr{A})$ is surjective, and $M_{n}(\mathscr{A})$ is generated (algebraically) by all diagonal unitaries and projections in $M_{n}(\tilde{\mathscr{A}})$.

4.8. RemarK. For any $n \geqq 2$, by Corollary 4.5 , one sees that there is no new path component created passing from $U_{n}(\mathscr{A})$ to a larger group $U_{n+1}(\mathscr{A})$ (under the natural embedding $\left.i_{n, n+1}\right)$ iff $\mathscr{G}_{n+1}(\mathscr{A})$ is connected.

4.9 EXAMPLES. The following two examples indicate that $\mathscr{G}_{n}(\mathscr{A})$ may not be connected in general as a subset of $\mathscr{P}\left(M_{n}(\tilde{\mathscr{A}})\right)$.

(1) Take $\mathscr{A}=C\left(S^{3}\right)$ and $n=2$. Let $u$ be the unitary in $M_{2}\left(C\left(S^{3}\right)\right)$ given by the homeomorphism $S^{3} \cong \mathrm{SU}(2)$. As pointed out in the second section, $u$ is not homotopic to a diagonal unitary. This implies that $u e_{2} u^{*}$ is not homotopic to $e_{2}$ in the Grassmann space of $M_{2}\left(C\left(S^{3}\right)\right)$. Thus, the unitary orbit $\mathscr{G}_{2}(\mathscr{A})$ of $e_{2}$ in $M_{2}\left(C\left(S^{3}\right)\right)$ is not connected. On the other hand, if $n \geqq 3$, then $\mathscr{G}_{n}(\mathscr{A})$ is connected; this follows from Corollary 4.6(1) and the fact that $\operatorname{tsr}\left(C\left(S^{3}\right)\right)=2$ which is equal to the integer part of $\frac{\operatorname{dim}(X)}{2}+1$ ([39]). In view of Remark 4.8, for any $n \geqq 3$ there is no new path component appearing in $U_{n}\left(C\left(S^{3}\right)\right)$ different from the path components in $U_{2}\left(C\left(S^{3}\right)\right)$, while, as a well known fact, new path component does arise when $U\left(C\left(S^{3}\right)\right)$ passes to $U_{2}\left(C\left(S^{2}\right)\right)$.

(2) Take $\mathscr{A}=C\left(S^{2}\right)$ and again $n=2$. Let $p\left(\right.$.) be a projection in $M_{2}\left(C\left(S^{2}\right)\right)$ defined by the homeomorphism $S^{2} \cong G_{1,2}$, where $G_{1,2}$ is the Grassmann space of all one-dimensional projections in $M_{2}(\mathrm{C})$. Explicitly, $p($.$) is the line bundle$

$$
p(z, \theta)=\left(\begin{array}{cc}
1+z & \theta\left(1-z^{2}\right)^{\frac{1}{2}} \\
\tilde{\theta}\left(1-z^{2}\right)^{\frac{1}{2}} & 1-z
\end{array}\right)
$$

where $(z, \theta)$ is the cylindrical coordinates with $-1 \leqq z \leqq 1$ and $\theta \in S^{1}$. It is well known thatthe Stiefel manifold $V_{1,2}$ of all unit vectors in $\mathrm{C}^{2}$ is homeomorphic to $S^{3}$, and the Hopf map $S^{3} \rightarrow S^{2}$ does not admit a cross-section. It follows that there is no unitary in $M_{2}\left(C\left(S^{2}\right)\right)$ such that $u p(.) u^{*}$ is a diagonal projection ([26]). It follows that $p\left(\right.$.) is not homotopic to $e_{2}$. Therefore, the unitary orbit of $e_{2}$ in $M_{2}\left(C\left(S^{2}\right)\right)$ is not connected. Due to the same reason as in Example 1 , if $n \geqq 3$, then $\mathscr{G}_{n}(\mathscr{A})$ is connected (because $\operatorname{tsr}\left(C\left(S^{2}\right)\right)=2$, again); and hence there is no new path component appearing in $U_{n}\left(C\left(S^{2}\right)\right)$ for $n \geqq 3$.

It follows from Corollary 4.6 that every unitary in the unitizations of both $C\left(S^{2}\right) \otimes \mathscr{K}$ and $C\left(S^{3}\right) \otimes \mathscr{K}$ and $C\left(S^{3}\right) \otimes \mathscr{K}$ can be approximated by a product of some symmetries and a unitary of the form $u_{2} \oplus\left(1-I_{2}\right)$, where $u_{2}$ is a unitary of $M_{2}\left(C\left(S^{2}\right)\right)$ or $M_{2}\left(C\left(S^{3}\right)\right)$, respectively.

4.10. Proposition. Assume that $\mathscr{A}$ is any $C^{*}$-algebra. Under the standard 
embedding of $M_{n}(\mathscr{A})$ into $M_{n+1}(\tilde{\mathscr{A}}), \mathscr{G}_{n}(\mathscr{A})$ is identified with a subset of $\mathscr{G}_{n+1}(\mathscr{A})$. Then $\mathscr{G}_{n}(\mathscr{A})$ is contained in a connected component of $\mathscr{G}_{n+1}(\mathscr{A})$.

Proof. Obviously, the projection $e_{n}$, regarded as an element in $M_{n+1}(\tilde{\mathscr{A}})$ under the embedding, is homotopic to $e_{n+1}$. In turn, in $\mathscr{G}_{n+1}(\mathscr{A}), e_{n+1}$ is clearly equivalent to, and hence homotopic to, any projection in $\mathscr{G}_{n}(\mathscr{A})$.

Before concluding this section, we would like to point out the following fact.

4.11. Proposition. Suppose that $\mathscr{A}$ is a $C^{*}$-algebra with property that unitarily equivalent projections are homotopic, and $p$ is a nontrivial projection of $\mathscr{A}$. Then there are symmetries $s_{1}, s_{2}, \ldots, s_{l}$ of $\tilde{\mathscr{A}}$ such that

$$
u=s_{1} s_{2} \ldots s_{1}\left(\begin{array}{cc}
u_{1} & 0 \\
0 & u_{2}
\end{array}\right) \text {, }
$$

where $u_{1} \in U(p \mathscr{A} p)$ and $u_{2} \in U((1-p) \tilde{\mathscr{A}}(1-p))$.

Proof. Use exactly the same proofs in this section and the section 2.

4.12. Remarks. The following three facts (1)-(3) are well known, where all subsets of $\mathscr{A}$ involved are equipped with the relative norm topology.

(1) The group of invertible elements of a unital $C^{*}$-algebra $\mathscr{A}$, denoted by $\mathrm{GL}(\mathscr{A})$, is homotopy equivalent to $U(\mathscr{A})$, since $U(\mathscr{A})$ is a homotopy retract of $\mathrm{GL}(\mathscr{A})$.

(2) The space of all nontrivial $(\neq 0,1)$ indempotents of $\mathscr{A}$ is homotopy equivalent to $\mathscr{P}(\mathscr{A})$.

(3) The space of symmetries of $\mathscr{A}$ is homotopy equivalent to the subspace

$$
\left\{x \in \mathrm{GL}(\mathscr{A}): x^{2}=1\right\} \text {. }
$$

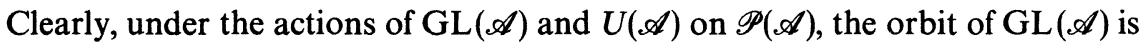
the space of all nontrivial idempotents, while the orbit of $U(\mathscr{A})$ is $\mathscr{P}(\mathscr{A})$. Thus, one can rephrase all results in the first section and the second section in terms of $\mathrm{GL}(\mathscr{A})$ and the space of non-trivial idempotents. This is left to the interested reader.

\section{REFERENCES}

1. C. A. Akemann, G. K. Pedersen and J. Tomiyama, Multipliers of $C^{*}$-algebras, J. Funct. Anal. 13 (1973), 277-301.

2. H. Araki, M.-S. B. Smith and L. Smith, On the homotopical significance of the type of von Neumann algebra factors, Comm. Math. Phys. 22 (1971), 71-88.

3. B. Blackadar, K-theory for operator algebras, Springer-Verlag, New York Berlin Heidelberg London Paris Tokyo, 1987.

4. B. Blackadar, O, Bratteli, G. A. Elliott and A. Kumjian, Reduction of real rank in inductive limits of $C^{*}$-algebras, preprint.

5. B. Blackadar, M. Dadarlat and M. Rфrdam, The real rank of inductive limits $C^{*}$-algebras, preprint. 
6. B. Blackadar and A. Kumjian, Skew products of relations and structure of simple $C^{*}$-algebras, Math. Z. 189 (1985), 55-63.

7. J. Bunce and J. Deddens, A family of simple $C^{*}$-algebras related to weighted shift operators, J. Funct. Anal. 19 (1975), 13-24.

8. M. Breuer, A generalization of Kuiper's theorem to factors of type $\mathrm{II}_{\infty}$, J. Math. Mech. 16 (1967), 917-925.

9. M. Breuer, On the homotopy type of the group of regular elements of semifinite von Neumann algebras, Math. Ann. 185 (1970), 61-74.

10. L. G. Brown, Stable isomorphism of hereditary subalgebras of $C^{*}$-algebras, Pacific J. Math. 71 (1977), 335-348.

11. L. G. Brown, Extensions of AF algebras: the projection lifting problem, Operator Algebra and Applications, Proc. Sympos. Pure Math. 38, part I, 1981, 175-176.

12. L. G. Brown, The rectifiable metric on the set of closed subspaces of Hilbert space, Trans. Amer. Math. Soc. (to appear).

13. L. G. Brown and G. K. Pedersen, $C^{*}$-algebras of real rank zero, J. Funct. Anal. 99 (1991), 131-149.

14. M.-D. Choi and G. A. Elliott, Density of the self-adjoint elements with finite spectrum in an irrational rotation $C^{*}$-algebra, Math. Scand. 67 (1990), 73-86.

15. J. Cuntz and N. Higson, Kuiper's theorem for Hilbert modules, Operator algebras and mathematical physics, Amer. Math. Soc., Vol. 62, Proceedings of a Summer conference, June 17-21, 1985.

16. J. Cuntz and W. Krieger, A class of $C^{*}$-algebras and topological Markov chains, Invent. Math. 56 (1980), 251-268.

17. J. Cuntz, K-theory for certain $C^{*}$-algebras, Ann. of Math. 131 (1981), 151-197.

18. J. Cuntz, Simple $C^{*}$-algebras generated by isometries, Comm. Math. Phys. 57 (1977), 173-185.

19. M. Dadarlat, G. Nagy, A. Nemethi and C. Pasnicu, Reduction of topological stable rank in inductive limits of $C^{*}$-algebras, Pacific J. Math. 153 (1992), 267-276.

20. E. G. Effros, Dimensions and $C^{*}$-algebras, CBMS Regional Conference Series in Mathematics A.M.S. 46 (1981).

21. E. G. Effros and J. Kaminker, Some homotopy and shape calculations for $C^{*}$-algebras, Group Representations, Ergodic Theory, Operator Algebras, And Mathematical Physics, MSRI Publication No. 6, Springer Verlag, New York (1987).

22. G. A. Elliott, Derivations of matroid $C^{*}$-algebras II, Ann. of Math. 100 (1974), 407-422.

23. G. A. Elliott, On the classification of $C^{*}$-algebras of real rank zero, preprint.

24. P. R. Halmos and S. Kakutani, Products of symmetries, Bull. Amer. Math. Soc. 64 (1958), 77-78.

25. K. R. Goodearl, Some notes on yet another family of simple $C^{*}$-algebras that sometimes have real rank zero, preprint.

26. K. Grove and G. K. Pedersen, Diagonalizing matrices over $C(X)$, J. Funct. Anal. 59(1984), 65-89.

27. G. C. Kasparov, Hilbert $C^{*}$-modules: theorems of Stinespring and Voiculescu, J. Operator Theory 4 (1980), 133-150.

28. N. H. Kuiper, The homotopy type of the unitary group of Hilbert space, Topology 3(1965), 19-30.

29. H. Lin, The simplicity of the quotient algebra $M(\mathscr{A}) / \mathscr{A}$ lf a simple $C^{*}$-algebra, Math. Scand. 65 (1989), 119-128.

30. H. Lin, Simple $C^{*}$-algebras with continuous scales and simple corona algebras, Proc. Amer. Math. Soc. 112 (1991), 871-880.

31. H. Lin, Generalized Weyl-von Neumann theorem, I, Internat. J. Math. 6 (1991), 725-739.

32. H. Lin and S. Zhang, On infinite simple $C^{*}$-algebras, J. Funct. Anal. 100 (1991), 2221-231.

33. R. V. Kadison and J. R. Ringrose, Fundamentals of the Theory of Operator Algebras Vol. I, II, Academic Press Inc., 1986.

34. J. A. Mingo, K-theory and multipliers of stable $C^{*}$-algebras, Trans. Amer. Math. Soc. 299 (1987), 397-411.

35. G. K. Pedersen, The linear span of projections in simple $C^{*}$-algebras, J. Operator Theory 4 (1980), 289-296. 
36. N. C. Phillips, Approximation by unitaries with finite spectrum in purely infinite $C^{*}$-algebras, J. Funct. Anal. (to appear).

37. N. C. Phillips, Simple $C^{*}$-algebras with the property weak (FU), Math. Scand. $69(1991), 127-151$.

38. N. C. Phillips, How many exponentials? Amer. J. Math. (to appear).

39. M. A. Rieffel, Dimension and stable rank in the K-theory of $C^{*}$-algebras, Proc. London Math. Soc. 46 (1983), 301-333.

40. M. A. Rieffel, The homotopy groups of the unitary groups of non-commutative tori, J. Operator Theory 17 (1987), 237-254.

41. M. A. Rieffel, The cancellation theorem for projective modules over irrational rotation $C^{*}$-algebras, Proc. London Math. Soc. 47 (1983), 285-302.

42. J. R. Ringrose, Exponential length and exponential rank in $C^{*}$-algebras, Proc. Royal Soc. Edinburgh (Sect. A) 121 (1992), 55-71.

43. H. Schröder, On the homotopy type of the regular group of a $W^{*}$-algebra, Math. Ann. 267 (1984), 271-277.

44. H. Schröder, The homotopy type of the regular group of a Banach algebra (a survey), preprint.

45. A. J.-L. Sheu, $A$ cancellation theorem for modules over the group $C^{*}$-algebras of certain nilpotent Lie groups, Canad. J. Math. 39 (1987), 365-427.

46. K. Thomsen, Non-stable $K$-theory for operator algebras, $K$-theory (in press).

47. K. Thomsen, Non-stable K-theory for operator algebras, II, preprint.

48. S. Zhang, Diagonalizing projections in the multiplier algebra and marices over a $C^{*}$-algebra, Pacific J. Math. 145 (1990), 181-200.

49. S. Zhang, A Riesz decomposition property and ideal structure of multiplier algebras, J. Operator Theory 24 (1990), 209-225.

50. S. Zhang, Certain $C^{*}$-algebras with real rank zero and their corona and multiplier algebras, Part I, Pacific J. Math. 155 (1992), 169-197.

51. S. Zhang, Certain $C^{*}$-algebras with real rank zero and their corona and multiplier algebras, Part II, $K$-theory 6 (1992), 1-27.

52. S. Zhang, $C^{*}$-algebras with real rank zero and the internal structure of their corona and multiplier algebras, Part III, Canad. J. Math. 62 (1990), 159-190.

53. S. Zhang, A property of purely infinite simple $C^{*}$-algebras, Proc. Amer. Math. Soc. 109 (1990), 717-720.

54. S. Zhang, On the homotopy type of the unitary group and the Grassmann space of purely infinite simple $C^{*}$-algebras, K-Theory (to appear).

55. S. Zhang, On the exponential rank and exponential length of $C^{*}$-algebras, J. Operator Theory (to appear).

56. S. Zhang, $K_{1}-$ groups, quasidiagonality and interpolation by multiplier projections, Trans. Amer. Math. Soc. 325 (1991), 793-818.

57. S. Zhang, On the structure of projections and ideals of corona algebras, Canad. J. Math. 41 (1989), 721-742.

58. S. Zhang, Exponential rank and exponential length of operators on Hilbert $C^{*}$-module, Ann. of Math. 137 (1993), 129-144.

59. S. Zhang, Matricial structure and homotopy type of simple $C^{*}$-algebras of real rank zero, J. Operator Theory 26 (1991), 283-312.

60. S. Zhang, Trivial $K_{1}-$ flow of AF algebras and finite von Neumann algebras, J. Funct. Anal. 92 (1990), 77-91.

61. S. Zhang, Rectifiable diameter of the Grassmann spaces of von Neumann algebras and certain $C^{*}$-algebras, preprint.

DEPARTMENT OF MATHEMATICAL SCIENCES

UNIVERSITY OF CINCINNATI

CINCINNATI, OHIO 45221-0025

U.S.A.

E-MAIL ADDRESS: Szhang@ucbeh.san.uc.edu 